\title{
Cambios en las hormonas sexuales en varones mayores de 50 años. Prevalencia de niveles bajos de testosterona y factores de riesgo
}

\author{
Martínez Jabaloyas JM, Queipo Zaragoza A*, Ferrandis Cortes C, Queipo Zaragoza JA**, \\ Gil Salom M, Chuan Nuez P.
}

\begin{abstract}
Servicio de Urología. *Laboratorio de Bioquímica Clínica. Hospital Clínico Universitario. Valencia. **Servicio de Urología. Hospital de Sagunto.
\end{abstract}

Actas Urol Esp. 2008;32(6):603-610

\section{RESUMEN}

CAMBIOS EN LAS HORMONAS SEXUALES EN VARONES MAYORES DE 50 AÑOS. PREVALENCIA DE NIVELES BAJOS DE TESTOSTERONA Y FACTORES DE RIESGO

Objetivos: Evaluar los cambios en las hormonas sexuales en una población masculina mayor de 50 años, conocer la frecuencia de hipogonadismo bioquímico y qué factores se relacionan con los niveles de testosterona.

Pacientes y método: Estudio transversal en 230 varones sanos mayores de 50 años. Se realizaron determinaciones de testosterona total, testosterona libre, testosterona biodisponible, sulfato de dehidroepiandrosterona, estradiol total y biodisponible, LH, FSH y prolactina y se averiguaron los antecedentes clínicos y sociodemográficos. Determinamos la frecuencia de hipogonadismo bioquímico. Analizamos los factores que podían influir en los niveles de testosterona total y libre mediante análisis uni y multivariante y realizamos un análisis de regresión logística para conocer los factores que pueden predecir el hipogonadismo bioquímico en base a la testosterona libre.

Resultados: A medida que incrementa la edad de los sujetos evaluados, se observan niveles significativamente más bajos (p<0,05) de los niveles de testosterona total $(0,6 \%$ anual), testosterona libre ( $1,3 \%$ anual), sulfato de dehidroepiandrosterona ( $1,8 \%$ anual) y estradiol biodisponible $(0,69 \%$ anual), mientras que se observó un incremento de la SHBG, la LH y la FSH (p>0,05).

La prevalencia de niveles bajos de testosterona total fue del 4,8\%, alcanzando el $24,8 \%$ cuando utilizamos como referencia la testosterona libre. La media de los niveles plasmáticos de testosterona total fue significativamente inferior en los varones con obesidad, diabetes mellitus o dislipemia, mientras que la de los niveles de testosterona libre fue menor en aquellos con con estilo de vida sedentario, bajo nivel de estudios, obesos o con diabetes mellitus. En el análisis multivariante, la edad, la diabetes y la obesidad se relacionaron inversamente con los niveles de testosterona total. Con la testosterona libre se relacionan los mismos factores además de la dislipemia. En el análisis de regresión logística multivariante para el hipogonadismo bioquímico, la edad, la obesidad y la diabetes fueron las variables con valor predictivo independiente.

Conclusión: A partir de los 50 años se produce un descenso significativo de testosterona total, testosterona libre, sulfato de dehidroepiandrosterona y estradiol biodisponible relacionado con la edad. La prevalencia de hipogonadismo bioquímico es mayor si utilizamos la testosterona libre. Los niveles de testosterona total se relacionan además de con la edad, con la diabetes y la obesidad, añadiéndose la dislipemia para la testosterona libre. La probabilidad presentar niveles bajos de testosterona libre aumenta con la edad y es mayor cuando existen antecedentes de diabetes mellitus y/u obesidad.

Palabras clave: Andropausia. Envejecimiento. Varón. Testosterona. Hipogonadismo. Hormonas sexuales.

\section{ABSTRACT}

CHANGES IN SEXUAL HORMONES IN A MALE POPULATION OVER 50 YEARS OF AGE. FREQUENCY OF LOW TESTOSTERONE LEVELS AND RISK FACTORS

Objectives: To investigate the changes in sexual hormones in a selected male population older than 50 years of age. To assess the frequency of biochemical hypogonadism and which factors are related to testosterone levels.

Patients and methods: A Cross-sectional study was carried out on 230 Spanish men older than 50 years of age. Blood tests were performed including: total testosterone, SHBG, calculated free testosterone, dehidroepiandrosterone sulfate, androstendione, estradiol, bioavailable estradiol, FSH, LH, and prolactin. Clinical and socio-demographic backgrounds were investigated.

The frequency of biochemical hypogonadism was established using total and free testosterone levels as diagnostic criteria. Factors that may influence testosterone levels were evaluated by univariate and multivariate statistical analysis, and a logistic regression model was used to determine which factors can predict biochemical hypogonadism according to free testosterone levels.

Results: Age was associated with a significant decrease $(\mathrm{p}<0.05)$ in total testosterone $(0.6 \%$ per year $)$, free testosterone $(1.3 \%$ per year $)$ dehidroepiandrosterone sulfate (1.8\% per year) and bioavailable estradiol (0.69\% per year). Moreover, an increase in SHBG, LH, and FSH was observed $(\mathrm{p}<0.05)$. According to total testosterone levels, $4.8 \%$ of the men were hypogonadal, whereas $24.8 \%$ were hypogonadal when free testosterone was considered. In the univariate analysis, obesity, diabetes mellitus and hyperlipemia were related to lower total testosterone levels, while free testosterone levels were lower in men with sedentary life, lower levels of education, obesity or diabetes mellitus. In the multivariate analysis age, diabetes mellitus and obesity were inversely related to total and free testosterone levels. Free testosterone was also inversely related to hyperlipemia. For biochemical hypogonadism, simple logistic regression analysis selected age, sedentary life, obesity and diabetes mellitus. In the multivariate analysis age, obesity and diabetes mellitus had significant independent prognostic value.

Conclusions: Starting from 50 years of age, a significant age-related decrease in total testosterone, free testosterone, dehidroepiandrosterone sulfate and bioavailable estradiol is observed. The frequency of biochemical hypogonadism is higher when free testosterone levels are used for diagnosis. Total testosterone levels were related to age, diabetes mellitus and obesity. Free testosterone was related to age, diabetes mellitus, obesity and hyperlipemia. The probability of suffering low free testosterone levels increases with age, diabetes mellitus and/or obesity. 
$\mathrm{E}^{1}$ progresivo declive androgénico del varón que envejece es una circunstancia conocida desde hace más de 60 años, cuando aparece el término "climaterio masculino" usado por Werner que lo describió como un complejo conjunto de síntomas que englobaba síntomas psicológicos (depresión, insomnio y pérdida de memoria y concentración), físicos (fatiga) y sexuales (menor vigor sexual) ${ }^{1}$.

En los últimos años observamos un aumento del interés en publicaciones científicas y en los congresos por los cambios hormonales que ocurren en el varón durante el envejecimiento. Las causas del nuevo interés por un viejo síndrome hay que buscarlas en el incremento notabilísimo de la esperanza de vida, de tal forma que en 1950, menos del 5\% de la población era mayor de 65 años, mientras que para el 2025 la proporción puede ser mayor del 15\%, superando en Europa el 20\%2. Además, se han desarrollado y comercializado nuevas formas de tratamiento para el déficit androgénico, más fisiológicas, cómodas de utilizar y con escasos efectos secundarios. Así pues, la finalidad es unir a la mayor esperanza de vida una mejora en la calidad de vida.

A diferencia de las mujeres, los hombres no sufren una caída brusca de las hormonas sexuales sino que los cambios hormonales en el varón anciano ocurren de una forma gradual, más sutil e inconstante. Aún así, la testosterona puede reducirse a la mitad desde los 30 años hasta los $80^{3,4}$. Pero no sólo existen cambios en los niveles de testosterona, sino también se han descrito cambios en la globulina trasportadora de hormona sexual (SHBG), en las hormonas del eje hipotálamo-hipofisario, y en los andrógenos suprarrenales, especialmente el sulfato de dehidroepiandrosterona (SDHEA) ${ }^{5,6}$.

Sin embargo, no existen estudios en nuestro país respecto a los cambios hormonales descritos. El objetivo de nuestro estudio es analizar los cambios en las hormonas sexuales que se producen en una población de varones sanos mayores de 50 años, conocer qué factores están relacionados con los niveles de testosterona total y libre, y conocer la prevalencia de niveles bajos de testosterona en dicha población así como conocer los factores que pueden predecir el hipogonadismo bioquímico, definido por los niveles de testosterona libre.

\section{MATERIAL Y MÉTODOS}

Estudio transversal en el que se incluyeron 230 varones mayores de 50 años (con un rango de 50 a 83 años), pertenecientes a las Áreas 3 y 4 de Salud de la Comunidad Valenciana, procedentes de Atención primaria y de las consultas externas de Urología. El único criterio de inclusión fue ser varón mayor de 50 años. Como criterios de exclusión: 1) padecer cualquier patología aguda o crónica inestable o invalidante como insuficiencia cardíaca, cardiopatía isquémica en el último año, enfermedad neoplásica, enfermedad cerebrovascular o osteoartrosis severa, 2) el uso de tratamientos concomitantes o cualquier enfermedad que pudiera modificar los niveles hormonales, 3) que el motivo de la consulta médica en ese momento fuera por alguna alteración en la esfera sexual.

Se obtuvieron los antecedentes sociodemográficos y clínicos: edad, estado civil, estudios, estilo de vida (activa o sedentaria), índice de masa corporal (IMC) hábito tabáquico, hábito enólico y morbilidad asociada (HTA, diabetes o dislipemia). Clasificamos a los pacientes como obesos cuando su IMC era igual o mayor a 30 según el Consenso SEEDO'2000 (Sociedad Española para el Estudio de la Obesidad) ${ }^{7}$. Se determinaron los niveles hormonales de testosterona total (Chemiluminescent Microparticle Immunoassay. ARCHITECT, Abbott Laboratories), testosterona libre, determinada por el método matemático descrito por Vermeulen ${ }^{8}$, S-DHEA (EIAgen DHEA$s$ de ADALTIS Italia S.p.A), androstendiona (radioinmunoensayo con el Kit de DSL-3800 ACTIVE Diagnosis Systems Laboratories, Inc. Coated-Tube Radioimmunoassay), estradiol (técnica de fluoroinmunoensayo con el Kit DELFIA FABRICADO POR Wallac Oy Turku, Finlandia) y su forma biodisponible (mediante el método matemático descrito por Södergard) ${ }^{9}$, LH (inmunoensayo quimioluminiscente con el Kit de biotrol Diagnostic para el autoanalizador MAGIA L) y FSH (inmunoensayo quimioluminiscente con el Kit de bioMérieux sa para el autoanalizador MAGIA L), prolactina (inmunoensayo quimiluminiscente con el Kit de bioMérieux s.a. para el autoanalizador de MAGIA L) y SHBG (fluoroinmunoensayo con el Kit de DELFIA fabricado por Wallac Oy Turku, Finlandia).

$\mathrm{Al}$ no disponer de valores de normalidad para la testosterona libre, se realizó un estudio previo utilizando 129 varones sanos de entre 20 y 45 años obteniendo las muestras de sangre del Banco de 
sangre del hospital Clínico Universitario de Valencia. En ellos determinamos los niveles de testosterona libre y establecimos los valores de normalidad para dicha población.

La información fue introducida en una base de datos y para el análisis estadístico se empleó el programa SPSS 12.0 para Windows.

Para analizar la relación de las diferentes hormonas con la edad se realizaron análisis de regresión lineal simple, determinando el coeficiente de correlación de Pearson.

Determinamos los niveles medios de testosterona total y libre para los grupos establecidos en base a los antecedentes clínicos y sociodemográficos y los comparamos mediante tests no paramétricos (U de Mann-Whitney y test de Kruskal-Wallis). Para analizar la influencia de las diferentes variables sobre los niveles de testosterona total y libre realizamos un estudio multivariante mediante un análisis de regresión lineal múltiple.

Establecimos la prevalencia de hipogonadismo bioquímico para la testosterona total y libre en base a los valores de normalidad que habíamos obtenidos. Finalmente realizamos un análisis de regresión logística uni y multivariante para conocer qué variables podían predecir niveles de testosterona libre por debajo del límite de normalidad. En todos los análisis estadísticos se estableció el nivel de significación en el 5\%.

\section{RESULTADOS}

El total de la población estudiada fue de 230 varones. La edad media fue de 66,3 \pm 8,2 años. Los antecedentes clínicos y sociodemográficos se describen en la Tabla 1. Entre los antecedentes destacar

Tabla 1. Antecedentes clínicos y modo de vida. Media de niveles plasmáticos de testosterona total y libre en base a los antecedentes y el modo de vida

\begin{tabular}{|c|c|c|c|c|c|c|}
\hline \multirow[b]{2}{*}{ Variables } & \multirow[b]{2}{*}{$\mathbf{N}$} & \multirow[b]{2}{*}{$\%$} & \multirow{2}{*}{$\begin{array}{l}\text { Testosterona } \\
\text { total (ng/ml) } \\
\text { X } \pm \text { DS }\end{array}$} & & \multirow{2}{*}{$\begin{array}{c}\text { Testosterona } \\
\text { libre (nmol/1) } \\
\text { X } \pm \text { DS }\end{array}$} & \multirow[b]{2}{*}{$\mathbf{P}$} \\
\hline & & & & $\mathbf{P}$ & & \\
\hline \multicolumn{7}{|l|}{ Tabaco } \\
\hline No & 168 & $(72,7)$ & $5,1 \pm 1,7$ & $0,487^{1}$ & $0,297 \pm 0,100$ & $0,396^{1}$ \\
\hline $\mathrm{Si}$ & 62 & $(22,3)$ & $5,3 \pm 1,8$ & & $0,304 \pm 0,092$ & \\
\hline \multicolumn{7}{|l|}{ Alcohol } \\
\hline No & 153 & $(66,5)$ & $5,1 \pm 1,6$ & & $0,297 \pm 0,091$ & \\
\hline$<40$ g/día & 66 & $(28,7)$ & $5,2 \pm 1,9$ & $0,838^{2}$ & $0,302 \pm 0,115$ & $0,948^{2}$ \\
\hline$>40$ g/día & 11 & $(4,8)$ & $5,1 \pm 1,6$ & & $0,300 \pm 0,081$ & \\
\hline \multicolumn{7}{|l|}{ Estudios } \\
\hline Sin estudios o primaries & 198 & $(86,1)$ & $5,1 \pm 1,7$ & $0,105^{1}$ & $0,293 \pm 0,111$ & $0,439^{1}$ \\
\hline Secundarios/universitarios & 32 & $(13,9)$ & $5,5, \pm 1,7$ & & $0,336 \pm 0,111$ & \\
\hline \multicolumn{7}{|l|}{ Vida en pareja } \\
\hline No & 33 & $(14,3)$ & $5,2 \pm 1,9$ & $0,831^{1}$ & $0,300 \pm 0,095$ & $0,439^{1}$ \\
\hline $\mathrm{Si}$ & 197 & $(85,7)$ & $5,1 \pm 1,7$ & & $0,299 \pm 0,098$ & \\
\hline \multicolumn{7}{|l|}{ Estilo de vida } \\
\hline Sedentario & 84 & $(36,5)$ & $5,1 \pm 1,7$ & $0,747^{1}$ & $0,279 \pm 0,097$ & $0,011^{1}$ \\
\hline Activo & 146 & $(63,5)$ & $5,2 \pm 1,7$ & & $0,310 \pm 0,096$ & \\
\hline \multicolumn{7}{|l|}{ Indice de Masa corporal } \\
\hline$<30$ (normal o sobrepeso) & 200 & $(86,9)$ & $5,2 \pm 1,7$ & $0,006^{1}$ & $0,307 \pm 0,098$ & $0,004^{1}$ \\
\hline$>30$ (obesidad) & 30 & $(13,1)$ & $4,4 \pm 1,6$ & & $0,252 \pm 0,078$ & \\
\hline \multicolumn{7}{|l|}{ Hipertensión } \\
\hline No & 164 & $(71,3)$ & $5,1 \pm 1,7$ & $0,831^{1}$ & $0,301 \pm 0,098$ & $0,439^{1}$ \\
\hline $\mathrm{Si}$ & 66 & $(28,7)$ & $5,0 \pm 1,7$ & & $0,294 \pm 0,097$ & \\
\hline \multicolumn{7}{|l|}{ Diabetes Mellitus } \\
\hline No & 126 & $(85,2)$ & $5,2 \pm 1,7$ & $0,010^{1}$ & $0,307 \pm 0,097$ & $0,003^{1}$ \\
\hline $\mathrm{Si}$ & 39 & $(14,8)$ & $4,4 \pm 1,6$ & & $0,299 \pm 0,089$ & \\
\hline \multicolumn{7}{|l|}{ Dislipemia } \\
\hline No & 108 & $(47,0)$ & $5,2 \pm 1,8$ & $0,014^{1}$ & $0,311 \pm 0,096$ & $0,405^{1}$ \\
\hline $\mathrm{Si}$ & 122 & $(53,0)$ & $5,1 \pm 1,7$ & & $0,294 \pm 0,097$ & \\
\hline
\end{tabular}

${ }^{1} \mathrm{U}$ Mann Withney. ${ }^{2}$ Kruskal.Wallis. 
que el 28,7\% eran hipertensos, el 14,8\% diabéticos y el 53\% presentaban alteración en el perfil lipídico y/o estaban en tratamiento con hipolipemiantes. El IMC medio fue de $26,7 \pm 2,7$, con un $13 \%$ de obesos (IMC $\geq 30$ ).

La edad mostró una relación inversa con los niveles de testosterona total, testosterona libre y DHEA-s $(\mathrm{p}<0,05)$ con una disminución anual del 0,6\%, 1,3\% y 1,8\% respectivamente (Figs. 1, 2 y 3). En el caso de la SHBG se observó una relación directa $(\mathrm{p}<0,05)$, con un incremento anual del 1,2\% (Fig. 4). La androstendiona, el estradiol y la prolactina no mostraron variación significativa con la edad. No obstante en la forma biodisponible del estradiol se observó un descenso significativo (p<0,05) del 0,69\% por año (Fig. 5). Como se observan en las Figuras 6 y 7 la LH y la FSH experimentaron un aumento anual del $1 \%$ y 1,2\% respectivamente $(\mathrm{p}<0,05)$.

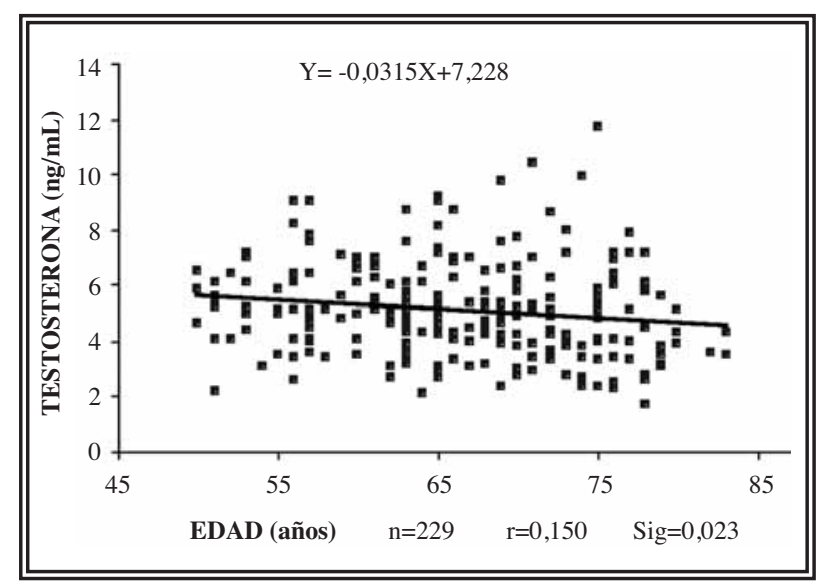

FIGURA 1. Relación entre los niveles de testosterona total y la edad (r=coeficiente de correlación de Pearson).

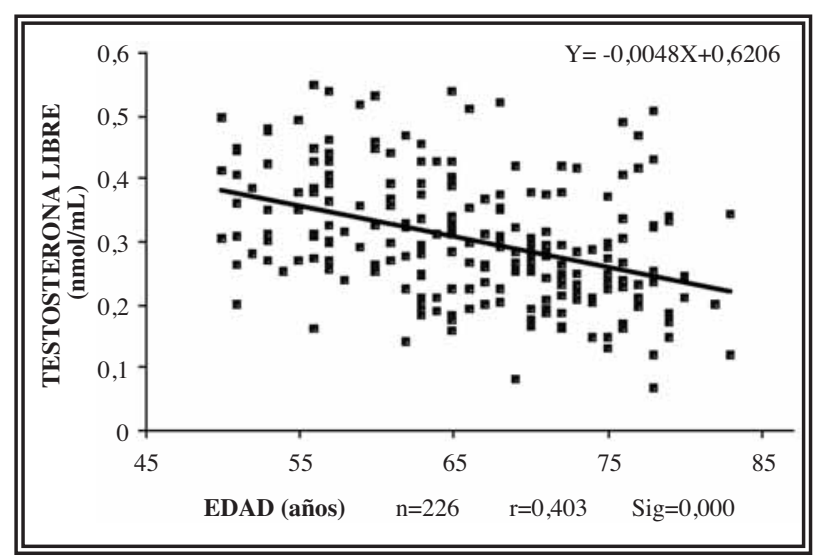

FIGURA 2. Relación entre los niveles de testosterona libre y la edad (r=coeficiente de correlación de Pearson).

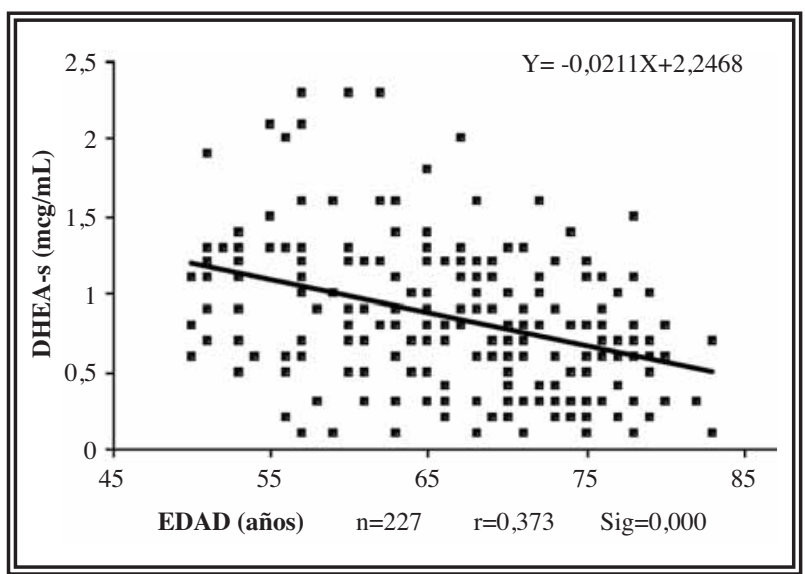

FIGURA 3. Relación entre los niveles de DHEA-s y la edad (r=coeficiente de correlación de Pearson).

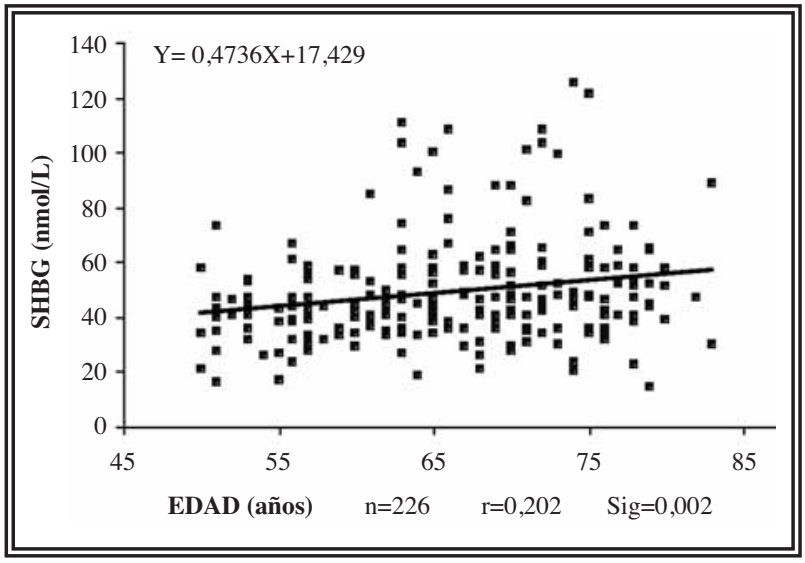

FIGURA 4. Relación entre los niveles de SHBG y la edad (r=coeficiente de correlación de Pearson).

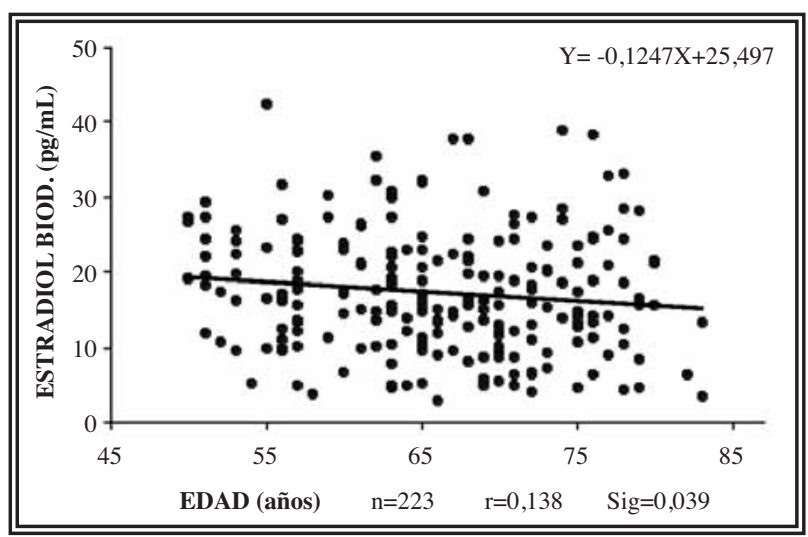

FIGURA 5. Relación entre los niveles de estradiol biodisponible y la edad (r=coeficiente de correlación de Pearson).

Los intervalos de normalidad obtenidos según la muestra de varones jóvenes sanos fueron de 2,5$10,2 \mathrm{ng} / \mathrm{mL}(5,13-17,38 \mathrm{nmol} / \mathrm{L})$ para la testosterona total y de $0,228-0,740 \mathrm{nmol} / \mathrm{L}$ para la testosterona libre. 


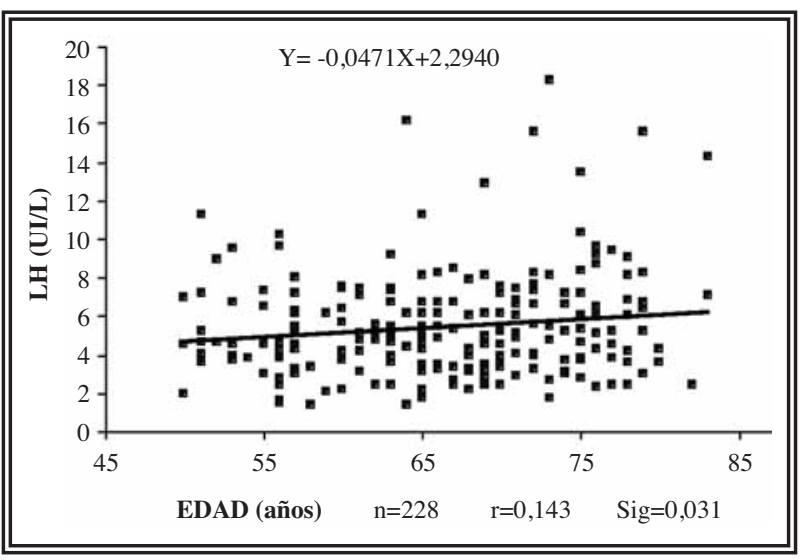

FIGURA 6. Relación entre los niveles de LH y la edad (r=coeficiente de correlación de Pearson).

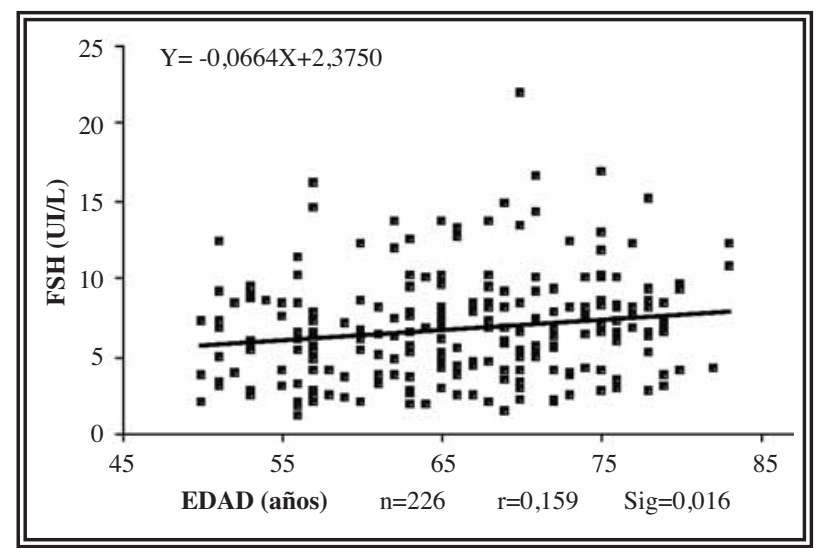

FIGURA 7. Relación entre los niveles de FSH y la edad. (r=coeficiente de correlación de Pearson).

En base a nuestros resultados, si utilizamos como criterio diagnóstico de hipogonadismo la testosterona total, la prevalencia de hipogonadismo bioquímico obtenida en nuestra población fue del $4,8 \%(11 / 220)$, mientras que si utilizamos la testosterona libre se eleva al 24,8\% (57/230).

Como apreciamos en el análisis univariante (Tabla 1) los niveles de testosterona total fueron significativamente menores en los varones afectos de obesidad, diabetes mellitus o dislipemia, mientras que los niveles medios de testosterona libre fueron menores en los varones con estilo de vida sedentario, bajo nivel de estudios, obesos o con diabetes mellitus. En el análisis multivariante (Tabla 2), la edad, la diabetes y la obesidad se relacionaron inversamente con los niveles de testosterona total, mientras que para la testosterona libre fueron las mismas variables además de la dislipemia.

Cuando analizamos qué variables pueden predecir un hipogonadismo bioquímico en base a los nive-
Tabla 2. Regresión lineal para la testosterona total y libre (variables dependientes) y edad, antecedentes clínicos y modo de vida (variables independientes)

\begin{tabular}{|c|c|c|c|c|}
\hline \multirow[b]{2}{*}{ Variables } & \multicolumn{3}{|c|}{ Testosterona total } & \multirow[b]{2}{*}{$\mathbf{r}^{*}$} \\
\hline & B & Beta $^{+}$ & $\mathbf{P}$ & \\
\hline Diabetes & $-0,705$ & $-0,147$ & 0,024 & 0,270 \\
\hline Obesidad (IMC $\geq 30$ ) & $-0,769$ & $-0,152$ & 0,020 & \\
\hline \multirow[t]{2}{*}{ Edad } & $-0,030$ & $-0,145$ & 0,026 & \\
\hline & \multicolumn{3}{|c|}{ Testosterona libre } & \\
\hline Variables & B & Beta $^{+}$ & $\mathbf{P}$ & $\mathbf{r}^{*}$ \\
\hline Edad & $-0,005$ & $-0,404$ & 0,000 & 0,494 \\
\hline Obesidad (IMC $\geq 30$ ) & $-0,047$ & $-0,163$ & 0,010 & \\
\hline Dislipemia & $-0,029$ & $-0,150$ & 0,016 & \\
\hline Diabetes & 0,034 & $-0,128$ & 0,042 & \\
\hline
\end{tabular}

+ Coeficiente estandarizado.

* Coeficiente de correlación múltiple.

les de testosterona libre, el análisis de regresión logística simple (Tabla 3) muestra que la edad, el sedentarismo, la obesidad y la diabetes fueron las variables que presentaron significación estadística. Sin embargo en el análisis multivariante únicamente la edad, la obesidad y la diabetes mostraron valor predictivo independiente.

\section{DISCUSIÓN}

El envejecimiento masculino se acompaña de progresivos cambios en prácticamente todo el eje endocrino. En contraste a la menopausia, es un proceso gradual con grandes variaciones individuales. En este estudio se corroboran, en una población española, los cambios hormonales previamente descritos durante el envejecimiento. Estos tienen lugar a nivel del sistema hipofisario, gonadal y adrenal, originando un descenso progresivo de los andrógenos y una elevación compensadora de las gonadotrofinas ${ }^{4,10,11}$. La testosterona plasmática desciende en nuestro estudio un $0,6 \%$ por año. Otros estudios transversales y longitudinales muestran descensos del 0,5 al $2 \%$ por año ${ }^{12}$, pero con grandes diferencias interindividuales. Además del descenso de la testosterona, existe un incremento de los niveles de globulina transportadora de hormona sexual. Esta proteína sanguínea es el mayor transportador sérico de testosterona, pero esta testosterona no es activa, con lo que el descenso de la producción de testosterona y el aumento de la producción de SHBG resulta, tal como observamos, que la testosterona libre desciende aún más que la testosterona total ${ }^{4}$. 
Tabla 3. Análisis de regresión logística para el hipogonadismo según la testosterona libre (eugonadismo/hipogonadismo) y los antecedentes sociodemográficos y clínicos (variables predictoras)

\begin{tabular}{|c|c|c|c|c|}
\hline \multirow[b]{2}{*}{ Variables } & \multicolumn{2}{|c|}{ Univariante } & \multicolumn{2}{|c|}{ Multivariante } \\
\hline & OR (IC 95\%)+ & $\mathbf{P}$ & OR (IC 95\%)+ & $\mathbf{P}$ \\
\hline Edad & $1,11(1,06-1,16)$ & 0,000 & $1,11(1,06-1,17)$ & 0,000 \\
\hline $\begin{array}{l}\text { Tabaco } \\
\text { No fumador } \\
\text { Fumador }\end{array}$ & $\begin{array}{c}1,00 \text { (ref.") } \\
0,85(0,42-1,69)\end{array}$ & 0,638 & & 0,299 \\
\hline $\begin{array}{l}\text { Alcohol } \\
\qquad \begin{array}{l}\text { No } \\
<40 \text { g/día } \\
>40 \text { g/día }\end{array}\end{array}$ & $\begin{array}{c}1,00 \text { (ref".) } \\
1,26(0,65-2,44) \\
1,23(0,31-4,89)\end{array}$ & $\begin{array}{l}0,779 \\
0,496 \\
0,767\end{array}$ & & 0,841 \\
\hline $\begin{array}{l}\text { Estudios } \\
\text { Sin estudios o primarios } \\
\text { Secundarios/universitarios }\end{array}$ & $\begin{array}{c}1,00 \text { (ref. ") } \\
1,07(0,45-2,54)\end{array}$ & 0,887 & & 0,106 \\
\hline $\begin{array}{l}\text { Vida en pareja } \\
\text { No } \\
\text { Si }\end{array}$ & $\begin{array}{c}1,00 \text { (ref. *) }^{*} \\
0,61(0,27-1,35)\end{array}$ & 0,221 & & 0,871 \\
\hline $\begin{array}{l}\text { Estilo de vida } \\
\text { Sedentaria } \\
\text { Activa }\end{array}$ & $\begin{array}{c}1,00 \text { (ref. *) } \\
0,42(0,23-0,78)\end{array}$ & 0,006 & & 0,473 \\
\hline $\begin{array}{l}\text { Índice de Masa Corporal } \\
\quad<30 \text { (normal o sobrepeso) } \\
\quad \geq 30 \text { (obesidad) }\end{array}$ & $\begin{array}{c}1,00 \text { (ref. ") } \\
2,96(1,32-6,64)\end{array}$ & 0,008 & $\begin{array}{c}1,00 \text { (ref. ") } \\
3,17(1,28-7,84)\end{array}$ & 0,013 \\
\hline $\begin{array}{l}\text { Hipertensión } \\
\text { No } \\
\text { Si }\end{array}$ & $\begin{array}{c}\left.1,00 \text { (ref. }^{*}\right) \\
0,96(0,49-1,87)\end{array}$ & 0,905 & & 0,883 \\
\hline $\begin{array}{l}\text { Diabetes } \\
\text { No } \\
\text { Si }\end{array}$ & $\begin{array}{c}1,00 \text { (ref. }^{*} \text { ) } \\
3,09(1,43-6,65)\end{array}$ & 0,004 & $\begin{array}{c}1,00 \text { (ref. *) }^{*} \\
2,86(1,24-6,61)\end{array}$ & 0,014 \\
\hline $\begin{array}{l}\text { Dislipemia } \\
\text { No } \\
\mathrm{Si}\end{array}$ & $\begin{array}{c}1,00 \text { (ref. *) } \\
1,89(0,99-3,57)\end{array}$ & 0,051 & & 0,051 \\
\hline
\end{tabular}

Este descenso de los niveles de testosterona, fundamentalmente de testosterona libre provoca, tal como observamos, que los niveles de LH estén incrementados en el varón añoso, aunque en ocasiones esto no ocurre o es insuficiente para compensar el descenso de testosterona, ya que con la edad se produce un descenso de la amplitud de los pulsos de la $\mathrm{LH}^{13}$. También se observa un incremento de la FSH, al disminuir con los años el número de células de Sertoli, así como la producción diaria de espermatozoides, siendo lo más frecuente el descenso del recuento y de la motilidad, manteniéndose la morfología normal ${ }^{14}$. El reflejo bioquímico es la disminución de los niveles de inhibina y la consiguiente elevación de la FSH.

La dehidroepiandrosterona es un andrógeno suprarrenal. El descenso de la dehidroepiandrosterona (DHEA) y su metabolito el sulfato de dehidroe- piandrosterona (S-DHEA), es un hallazgo constante en la edad avanzada. En la $5^{\text {a }}$ década de la vida los niveles de DHEA son un 30\% inferiores que en los hombres menores de 30 años ${ }^{15}$, con un descenso medio del $22 \%$ por década de vida y aunque sólo se han descrito pequeñas interacciones entre la sintomatología del varón que envejece y los niveles séricos de S-DHEA sí que se ha relacionado con cierta afectación de la función sexual ${ }^{16}$.

En el caso del estradiol existe más discrepancia entre diversos autores, de forma que se ha descrito un descenso del estradiol total ${ }^{17}$. Nosotros, como en otros estudios ${ }^{4,16}$ no observamos una correlación entre los niveles del mismo y la edad. En este estudio, así como en otros anteriores, sí se ha encontrado una relación estadísticamente significativa entre la edad y la fracción biodisponible del estradiol, no así para el total ${ }^{18}$. 
En nuestro estudio, la edad es un factor determinante en los niveles de testosterona y existe un incremento del hipogonadismo directamente relacionado con los años como previamente se ha descrito $^{10}$. Se observa una mayor prevalencia de hipogonadismo bioquímico cuando utilizamos la testosterona libre como indicador en lugar de la testosterona total. Además de la edad, otros factores pueden relacionarse con niveles más bajos de testosterona como la diabetes y la obesidad, añadiéndose la dislipemia para la testosterona libre. Nosotros definimos el hipogonadismo bioquímico en base a los niveles de testosterona libre, ya que si es la fracción plasmática realmente activa será la que cuando esté alterada pueda producir alteraciones en la fisiología del individuo. Sin embargo existe discordancia en las distintas publicaciones a la hora de evaluar los resultados en base a la testosterona libre, biodisponible o total. Cuando analizamos qué variables pueden relacionarse con niveles de testosterona libre por debajo de la normalidad observamos que la probabilidad de presentar hipogonadismo bioquímico es mayor en los pacientes obesos y en los diabéticos. Nuestro estudio confirma resultados previos en los que se ha observado una relación inversa entre los niveles de testosterona y el $\mathrm{IMC}^{17,19}$, de tal forma que el IMC puede ayudar a la identificación de hombres con probable deficiencia androgénica ${ }^{20}$. Los niveles de testosterona se relacionan de forma inversa con la masa grasa del organismo ${ }^{21}$. Los estudios de correlación no pueden aclarar si la obesidad induce niveles bajos de testosterona o si una gran cantidad de grasa visceral puede provocar un descenso de los niveles de testosterona, pero estudios prospectivos han mostrado que niveles bajos de andrógenos predicen aumento de la adiposidad en los varones ${ }^{22,23}$. También se ha propuesto que la relación entre testosterona y obesidad puede ser bidireccional $^{24}$, aunque el tratamiento sustitutivo con testosterona provoca un descenso en la grasa visceral $^{25}$, por lo que parece que los niveles de testosterona tendrían un papel importante en el incremento de la grasa.

Como vemos en nuestro estudio, existe una relación inversa entre la diabetes mellitus y los niveles de testosterona total y libre. Además, la probabilidad de presentar hipogonadismo es mayor en los varones diabéticos, lo que coincide con previos reportes en los que los varones con diabetes melli- tus tipo 2 presentan con elevada frecuencia hipogonadismo ${ }^{26,27}$. Destaca sobre todo que la relación entre diabetes e hipogonadismo es independiente de la obesidad. Se ha intentado explicar los bajos niveles de testosterona en pacientes obesos en base a los niveles más bajos de SHBG que estos tendrían, debido a un estado de hiperinsulinemia ${ }^{28}$. Sin embargo observamos que la diabetes y la obesidad se relacionan con niveles más bajos tanto de testosterona total como de libre, mientras que si se debiera a un descenso de los niveles de SHBG existiría relación solamente con los niveles de testosterona total. Además se ha descrito que las concentraciones de testosterona libre se relacionan inversamente con el IMC en los pacientes con diabetes tipo $2^{26,29}$.

Observamos que la alteración en el perfil lipídico se relaciona con los niveles de testosterona libre pero no con la total. Sin embargo, al estratificar a los pacientes en base a que la testosterona libre sea normal o baja no se observa relación con la dislipemia. En este aspecto se ha descrito que existe relación entre niveles de triglicéridos y testosterona ${ }^{23}$ y que el tratamiento sustitutivo con testosterona puede ser beneficioso frente a la hipercolesterole$\mathrm{mia}^{30}$.

Se ha encontrado relación entre el hipogonadismo y la mortalidad secundaria a enfermedades cardiovasculares $^{31}$. También se ha descrito una relación inversa entre los niveles de presión arterial y la testosterona ${ }^{23}$. Sin embargo en nuestro estudio no observamos que la hipertensión arterial se relacionara con los niveles de testosterona libre ni total.

\section{CONCLUSIONES}

A partir de los 50 años se produce un descenso de los niveles de testosterona, especialmente de la testosterona libre. Además se produce un descenso importante de los niveles de DHEA-S. La prevalencia de hipogonadismo bioquímico, si utilizamos la testosterona libre, alcanza al $25 \%$ de los varones mayores de 50 años. Los niveles de testosterona se relacionan, además de con la edad, con la diabetes y la obesidad, además de la dislipemia para la testosterona libre. La probabilidad presentar niveles bajos de testosterona libre aumenta con la edad y es mayor cuando existen antecedentes de diabetes mellitus u obesidad. 


\section{REFERENCIAS}

1. Werner AA. The male climacteric. JAMA 1939; 112: 1441-1443.

2. Schulman CC. The aging male: a challenge for urologists. CurR Opin Urol. 2000;10(4):337-345.

3. Lamberts SW, van den Beld AW, van der Lely AJ. The endocrinology of aging. Science. 1997;278(5337):419-424.

4. Gray A, Feldman HA, McKinlay JB, Longcope C. Age, disease, and changing sex hormone levels in middle-aged men: results of the Massachusetts Male Aging Study. J Clin Endocrinol Metab. 1991;73(5):1016-1025.

5 . Herbert J. The age of dehydroepiandrosterone. Lancet. 1995; 345 (8959): 1193-1194.

6. Feldman HA, Longcope C, Derby CA, Johannes CB, Araujo AB, Coviello $\mathrm{AD}$, et al. Age trends in the level of serum testosterone and other hormones in middle-aged men: longitudinal results from the Massachusetts male aging study. J Clin Endocrinol Metab. 2002;87(2):589-598.

7. Sociedad Española para el Estudio de la Obesidad (SEEDO). Consenso SEEDO'2000 para la evaluación del sobrepeso y la obesidad y el establecimiento de criterios pde intervención terapéutica. Med Clin (Barc) 2000; 115: 487-597.

8. Vermeulen A, Verdonck L, Kaufman JM A critical evaluation of simple methods for the estimation of free testosterone in serum. J Clin Endocrinol Metab. 1999;84(10):3666-3672.

9. Södergård R, Bäckström $T$, Shanbhag V, Carstensen $H$. Calculation of free and bound fractions of testosterone and estradiol-17 beta to human plasma proteins at body temperature. J Steroid Biochem. 1982;16(6):801-810.

10. Harman SM, Metter EJ, Tobin JD, Pearson J, Blackman MR; Baltimore Longitudinal Study of Aging. Longitudinal effects of aging on serum total and free testosterone levels in healthy men. Baltimore Longitudinal Study of Aging. J Clin Endocrinol Metab. 2001;86(2):724-731.

11. Elmlinger MW, Dengler T, Weinstock C, Kuehnel W. Endocrine alterations in the aging male. Clin Chem Lab Med. 2003;41(7): 934-941.

12. Seftel AD. Male hypogonadism. Part I: Epidemiology of hypogonadism. Int $\mathrm{J}$ of Impotence Research. 2006;18(2):115-120.

13. Vermeulen A, Kaufman JM. Diagnosis of hypogonadism in the aging male. Aging Male. 2002;5(3):170-176.

14. Weidner W, Diemer T, Bergmann M. Infertility in the aging male. In Lunenfeld B, Gooren L, eds. Texbook of Men's Health. London: The Parthenon Publishing Group. 2002: 97-103.

15. Herbert J. The age of dehydroepiandrosterone. Lancet. 1995; 345(8959): 1193-1194.

16. Ponholzer A, Plas E, Schatzl G, Jungwirth A, Madersbacher S; Austrian Society of Urology. Association of DHEA-S and estradiol serum levels to symptoms of aging men. Aging Male. 2002;5(4):233-238.

17. Van den Beld AW, de Jong FH, Grobbee DE, Pols HA, Lamberts SW. Measures of bioavailable serum testosterone and estradiol and their relationships with muscle strength, bone density, and body composition in elderly men. J Clin Endocrinol Metab. 2000;85(9):3276-3282.

18. Vermeulen A, Kaufman JM, Goemaere S, van Pottelberg I. Estradiol in elderly men. Aging male. 2002;5(2):98-102.
19. Vermeulen A, Goemaere S, Kaufman JM. Testosterone, body composition and aging. J Endocrinol Invest. 1999;22(5 Suppl): 110-116.

20. Kratzik CW, Reiter WJ, Riedl AM, Lunglmayr G, Brandstätter $\mathrm{N}$, Rücklinger E, et al. Hormone profiles, body mass index and aging male symptoms: results of the Androx Vienna Municipality study. Aging male. 2004;7(3):188-196.

21. Couillard C, Gagnon J, Bergeron J, Leon AS, Rao DC, Skinner JS, et al. Contribution of body fatness and adipose tissue distribution to the age variation in plasma steroid hormone concentrations in men: the HERITAGE family study. J Clin Endocrinol Metab. 2000;85(3):1026-1031.

22. Khaw KT, Barret-Connor E. Lower endogenous androgens predict central adiposity in men. Ann Epidemiol. 1992;2(5):675682 .

23. Zmuda JM, Cauley JA, Kriska A, Glynn NW, Gutai JP, Kuller LH. Longitudinal relation between endogenous testosterone and cardiovascular risk factors en middle-ageed men: a 13 years follow-up of former Multiple Risk Factor Intervention Trial participants. Am J Epidemiol. 1997;146(8):609-617.

24. Pitteloud N, Hardin M, Dwyer AA, Valassi E, Yialamas M, Elahi $\mathrm{D}$, et al. Increasing insulin resistance is associated with a decrease in Leydig cell testosterone secretion in men. J Clin Endocrinol Metab. 2005;90(5):2636-2641.

25. Mårin P, Holmäng S, Gustafsson C, Jönsson L, Kvist H, Elander A, et al. Androgen treatment of abdominally obese men. Int J Obesity Res. 1993;1(4):245-251.

26. Dhinda S, Prabhakar, S, Sethi M, Bandyopadhyay A, Chaudhuri A, Dandona P. Frequent occurrence of hypogonadotropic hypogonadism in type 2 diabetes. J Clin Endoncrinol Metab. 2004;89(11):5462-5468.

27. Mulligan T, Frick MF, Zuraw QC, Stemhagen A, McWhirter C. Prevalence of hypogonadism in males aged at least 45 years: the HIM study. Int J Clin Pract. 2006;60(7):762-769.

28. Kaufman JM, Vermeulen A. Declining gonadal function in elderly men. Baillières Clin Endocrinol Metab. 1997;11(2):289-298.

29. Tomar R, Dhinda S, Chaudhuri A, Mohanty P, Garg R, Dandona $\mathrm{P}$. Contrasting testosterone concentrations in type 1 and tipe 2 diabetes. Diabetes Care. 2006;29(5):1120-1122.

30. Kapoor D, Goodwin E, Channer KS, Jones TH. Testosterone replacement therapy improves insulin resistance, glycaemic control, visceral adiposity and hypercholesterolaemia in hypogonadal men with type 2 diabetes. Eur J Endocrinol. 2006;154 (6):899-906.

31. Khaw KT, Dowsett M, Folkerd E, Bingham S, Wareham N, Luben R, et al. Endogenous testosterone and mortality due to all causes, cardiovascular disease, and cancer in men: European prospective investigation into cancer in Norfolk (EPIC-Norfolk) Prospective Population Study. Circulation. 2007;116(23):2694-2701.

Correspondencia autor: Dr. J.M. Martínez Jabaloyas Servicio de Urología. Hospital Clínico Universitario. Avda. Blasco Ibáñez, 17 - 46010 Valencia. Tel.: 963862600 E-mail autor: jaba@pulso.com

Información artículo: Original - Andrología

Trabajo recibido: enero 2008

Trabajo aceptado: marzo 2008 